\title{
Theoretical investigation on two-dimensional monofluorinating phenethylammonium perovskite
}

\author{
Guomin Sui ${ }^{1}$, Guang Yang ${ }^{1}$, Bao Zhang ${ }^{2}$, and Yaqing Feng ${ }^{3}$ \\ ${ }^{1}$ Tianjin University \\ ${ }^{2}$ Tian Jin University \\ ${ }^{3}$ Tianjin Univeristy
}

February 2, 2022

\begin{abstract}
Detailed theoretical investigations on their structural and electronic properties of two-dimensional (2D) monofluorinating phenethylammonium perovskite are required to deeply understand the origin of the crystal structural and optoelectronic differences caused by organic spacers. Herein, formation energy, non-covalent interaction, band structure, exciton binding energy, carrier mobility and optical absorption spectra of $2 \mathrm{D}$ monofluorinating phenethylammonium lead iodide perovskite were calculated using density functional theory. Results show that steric effect leads to the orientational disorder of spacers in ortho-fluorinating phenethylammonium perovskite and weak interaction leads to the herringbone configuration of spacers in meta-fluorinating phenethylammonium perovskite. Exciton binding energy significantly influences the short circuit current density (JSC) of fluorophenethylammonium-based 2D perovskites, which can well explain the experimental trends of JSC. Formation energy analysis of perovskites with the same chemical components but different crystal structures and exciton binding energy calculation can be additional strategies in high-throughput screening for high-performance 2D perovskites.
\end{abstract}

\section{Hosted file}

Main document.docx available at https://authorea.com/users/451221/articles/555055theoretical-investigation-on-two-dimensional-monofluorinating-phenethylammoniumperovskite

\section{Hosted file}

Table.docx available at https://authorea.com/users/451221/articles/555055-theoreticalinvestigation-on-two-dimensional-monofluorinating-phenethylammonium-perovskite 